\title{
High Volume Adrenalin Solution Infiltration for Surgical Treatment of Gynaecomastia
}

\author{
Erkan Orhan ${ }^{1}$
}

${ }^{1}$ Gaziantep University, Department of Plastic Surgery, Gaziantep, Turkey

Correspondence:

Erkan Orhan

Address: Gaziantep University, Department of Plastic Surgery, Gaziantep, Turkey

Email: eorhan@yahoo.com

Received: 27.08 .2018 ,

Accepted: 07.12.2018

https://doi.org/10.5799/jcei/4001

\begin{abstract}
Objectives: Bleeding is an important problem in the surgical treatment of gynecomastia. The most implemented method to decrease the amount of bleeding is adrenaline infiltration, but adrenalin is mostly infiltrated with the fluid in the volume less than $250 \mathrm{cc}$ in the clinic. It is expected that adrenaline will accumulate more in the tissue and thus, exhibit more bleedingreducing effect by increasing the fluid volume used in the infiltration. In this study, the aim is to reveal the effect of increasing the amount of adrenaline in tissues by infiltrating a highervolume solution without increasing the adrenaline concentration in patients who have received gynecomastia treatment by an open surgery on the postoperative blood loss and surgical complications in patients.
\end{abstract}

Materials and Methods: Patients who had undergone open gynecomastia surgery between 2011 and 2017 were retrospectively examined. Patients not subjected to infiltration were described as Group 1 (n:9), patients infiltrated with adrenalin $100 \mathrm{cc}$ were described as Group 2 (n:13), and patients infiltrated with adrenalin $500 \mathrm{cc}$ were described as Group 3 (n:23).

Results: The average decrease in the hemoglobin level (bleeding) after the operation was calculated to be $2.00 \pm 0.37$ in Group 1, $1.5 \pm 0.4$ in Group 2, $0.7 \pm 0.30$ in Group 3 and the differences between groups were statistically significant $(p<0.001)$.

Conclusion: The infiltration of adrenaline in the same concentration with a fluid of a higher volume decreases blood loss after the gynecomastia surgery operation.

Keywords: gynecomastia, blood loss, infitration

\section{INTRODUCTION \\ Gynecomastia is described as the} enlargement of male breast tissue [1]. It is a common condition that can be determined at various levels in $40-55 \%$ of males [2,3]. In addition to being able to occur due to the benign proliferation of the breast glandular tissue, gynecomastia can occur due to the increased adipose tissue amount on the pectoral muscle, and this latter condition is called pseudogynecomastia. In most patients, these two conditions coexist [1]. Gynecomastia can occur due to hormonal imbalances during puberty, medications, malignancies, and chronic diseases but it is mostly idiopathic $[1,2,4]$.

Although gynecomastia is mostly bilateral, it can be observed to be unilateral or asymmetrical [3]. Simon's classification is mostly used in clinical classification.
According to this classification, gynecomastia is classified as Grade 1; minor breast enlargement without skin sagging, Grade 2a; moderate level breast enlargement without skin sagging, Grade 2b; moderate level breast enlargement with minor skin sagging, and Grade 3; advanced level breast enlargement with skin sagging [5].

Gynecomastia patients want to be treated due to complaints such as anxiety, lack of self-confidence and embarrassment, functional problems, psychosocial discomfort and fear of malignancy development [6,7]. In gynecomastia treatment, there are follow-up, medical treatment, and surgical treatment options. Surgical treatment is the most appropriate treatment for cases that continue for a long time and do not respond to medical treatment [3]. 
Bleeding, in particular, is an important problem in the surgical treatment of gynecomastia. In aesthetic surgery, bleeding problems are experienced in many procedures such as reduction mammoplasty, rhinoplasty, facelift, and liposuction, apart from gynecomastia, and the infiltration of adrenaline-containing solutions together with local anesthetics is performed to reduce the amount of bleeding and provide postoperative analgesia $[7,8]$. Adrenaline acts as a vasoconstrictor on tissues and reduces the amount of bleeding; however, especially when adrenaline solutions in a concentration higher than 1:100 000 are used, this vasoconstrictor effect leads to skin necrosis by reducing the tissue oxygen pressure and increases the risk of wound site infections $[7,8]$. Furthermore, in the postoperative period, bleeding may occur due to the termination of the vasoconstrictor effect that the high concentration of adrenaline has created on blood vessels, and this is called rebound bleeding. Rebound bleeding increases the risk of hematoma, infection, and failure of wound healing in the postoperative period $[9,10]$. Due to these side effects, infiltration solutions containing adrenaline concentration at ratios varying between 1:100 000 and 1:1000 000 are used in aesthetic procedures $[2,8,11]$.

In this study, the aim is to reveal the effect of increasing the amount of adrenaline in tissues by infiltrating a highervolume solution without increasing the adrenaline concentration in patients who have received gynecomastia treatment by an open surgery on the postoperative blood loss and surgical complications in patients.

\section{MATERIALS AND METHODS}

In this study, patients who had undergone open surgery for Simon Grade 2a and 2b gynecomastia between July 2011 and December 2017 were retrospectively examined. All patients' ages, preoperative hemoglobin $(\mathrm{Hb})$ and hematocrit (Hct) values, breast tissue weight removed during the surgery, whether infiltration was performed and if performed, the adrenaline concentration used, the hb and hct levels in the postoperative 24th hour, the amount of fluid coming from the drain in the postoperative 24-hour, length of hospitalization, and complications developing in patients were recorded. Patients with a bleeding problem, coagulation disorder, impairment in the prothrombin time (PT) and partial thromboplastin time (PTT) tests, and receiving anticoagulant and antithrombotic treatment were not included in the study. All patients were prohibited from taking aspirin, ibuprofen, alcohol, vitamin E, and smoking cigarettes during the preoperative and postoperative 10-day period.

\section{Surgical Technique}

The infiltration of adrenaline-containing fluid was not performed in the operations of patients who received gynecomastia treatment by an open surgery between July 2011 and November 2013 in our clinic. As a result of the significant blood loss in these patients, since November 2013, a solution containing adrenaline at the ratio of 1:1000 000 has been infiltrated in a volume of $100 \mathrm{cc}$ to each breast in the operations of patients receiving gynecomastia treatment by open surgery. In consequence of continuing blood loss, since September 2015, the solution containing adrenaline at the ratio of 1:1000 000 has started to be infiltrated in a volume of $500 \mathrm{cc}$ to each breast [12]. Patients in whom infiltration was not performed were identified as Group 1, patients in whom $100 \mathrm{cc}$ infiltration was performed were identified as Group 2, and patients in whom $500 \mathrm{cc}$ infiltration was performed were identified as Group 3.

All procedures were performed under general anesthesia. One hour before anesthesia, single dose prophylactic antibiotic treatment was applied to all patients [13]. The infiltration solution was prepared by mixing $1000 \mathrm{ml}$ of Ringer's lactate, $20 \mathrm{ml}$ of $2 \%$ lidocaine, and 1 ampule of 1:1000 epinephrine [9]. A stab incision was performed 2-3 $\mathrm{cm}$ below the inferior margin of the areola. With a standard infiltration cannula $3 \mathrm{~mm}$ in diameter and $20 \mathrm{~cm}$ in length, $100 \mathrm{cc}$ solution infiltration was performed to each breast in Group 2, and $500 \mathrm{cc}$ solution infiltration was performed to each breast in Group 3. The vasoconstriction effect of adrenaline was waited for 15-20 minutes following the infiltration, and then, the operation was started [14]. In patients undergoing open surgery only, incision of a semilunar shape was made from the lower $1 / 2$ of the nippleareola complex, and the breast gland and adipose tissues were excised. Appropriate bleeding control was performed after the excision. Then, a drain was placed in each breast. The incisions were sutured using 5-0 monocryl suture (Ethicon, Inc., Somerville, New Jersey). After dressing, patients were put on a 9-inch compression dressing. All patients were kept in hospital for at least 24 hours. The blood $\mathrm{hb}$ and hct values and the amount of fluid in the drains in the postoperative 24 th hour were measured. When the drainage volume was observed to be less than $20 \mathrm{cc}$ in 24 hours, the drains were pulled, and patients were discharged.

\section{RESULTS}

A total of 45 patients with Simon's $2 \mathrm{a}$ and $2 \mathrm{~b}$ gynecomastia were operated by open surgery in our clinic between July 2011 and December 2017. Surgical treatment was applied to 9 of the patients without infiltration (Group 1 ), to 13 of the patients by infiltrating $100 \mathrm{cc}$ adrenaline solution (Group 2), and to 23 of the patients by infiltrating $500 \mathrm{cc}$ adrenaline solution (Group 3) (Table 1).

There was no statistically significant difference between the groups in terms of the patient's age, amount of the tissue excised, and preoperative $\mathrm{hb}$ values $(\mathrm{p}=0.858, \mathrm{p}=0.331$, $\mathrm{p}=0.710)$ (Table 1,2). The mean amount of a decrease in the postoperative hb value was measured as $2.00 \pm 0.37 \mathrm{~g} / \mathrm{dl}$ in Group 1, $1.5 \pm 0.44 \mathrm{~g} / \mathrm{dl}$ in Group 2, and $0.7 \pm 0.30 \mathrm{~g} / \mathrm{dl}$ in Group 3. Upon comparing the groups in terms of the amount of bleeding, the amount of bleeding in Group 2 and Group 3 was found to be statistically significantly lower than 
High Volume Adrenalin Solution For Gynaecomastia

Table 1. Demographical and clinical characteristics

\begin{tabular}{lcccc}
\hline & Group 1 $(\mathbf{n}=\mathbf{9})$ & Group 2 $(\mathbf{n}=\mathbf{1 3})$ & Group 3 (n=23) & p-value \\
\hline Age (years) & $25.1 \pm 5.0$ & $24.5 \pm 4.2$ & $24.1 \pm 4.5$ & $0.858{ }^{\dagger}$ \\
\hline Resection of tissue & $203.3 \pm 41.2$ & $198.5 \pm 36.0$ & $216.5 \pm 35.0$ & $0.3311^{\dagger}$ \\
\hline Hospitalization (days) & $2(1-2)^{\mathrm{a}, \mathrm{b}}$ & $1(1-2)^{\mathrm{a}, \mathrm{c}}$ & $1(1-1)^{\mathrm{b}, \mathrm{c}}$ & $<\mathbf{0 0 1 \ddagger}$ \\
\hline Amount of drain & $40(10-50)^{\mathrm{a}, \mathrm{b}}$ & $20(0-50)^{\mathrm{a}, \mathrm{c}}$ & $0(0-15)^{\mathrm{b}, \mathrm{c}}$ & $<\mathbf{0 0 1 \ddagger}$ \\
\hline Complication & $0(0.0 \%)$ & $2(15.4 \%)$ & $0(0.0 \%)$ & $\mathbf{0 . 0 7 4 ף}$
\end{tabular}

† One-Way ANOVA, ₹ Kruskal Wallis test, ๆ Likelihood ratio test, a: Group 1 vs Group 2 ( $<<0.05)$, b: Group 1 vs Group 3 ( $<<0.001$ ), c: Group 2 vs Group $3(p<0.05)$.

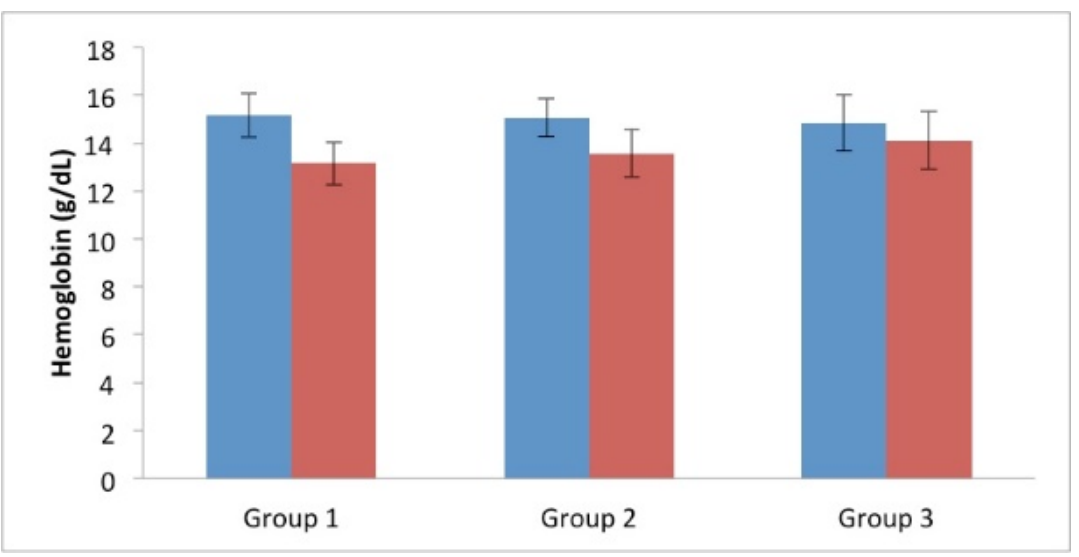

Figure 1. Preoperatif(blue) and postoperatif(red) hb level in groups

Table 2. Pre- and post-op hemoglobin measurements regarding for study groups

\begin{tabular}{lccccc}
\hline & $\mathbf{n}$ & Pre-op & Post-op & p-value $\boldsymbol{~}$ & Difference \\
\hline Group 1 & 9 & $15.1 \pm 0.92$ & $13.1 \pm 0.91$ & $<0.001$ & $-2.00 \pm 0.37^{\mathrm{a}, \mathrm{b}}$ \\
\hline Group 2 & 13 & $15.1 \pm 0.79$ & $13.6 \pm 0.98$ & $<0.001$ & $-1.5 \pm 0.44^{\mathrm{a}, \mathrm{c}}$ \\
\hline Group 3 & 23 & $14.8 \pm 1.17$ & $14.1 \pm 1.20$ & $<0.001$ & $-0.7 \pm 0.30^{\mathrm{b}, \mathrm{c}}$ \\
\hline p-value $\mathbf{}$ & & 0.710 & 0.079 & & $<0.001$ \\
\hline
\end{tabular}

Data were shown as mean \pm standard deviation, + The comparisons between pre- and post-op within each group, Paired t-test, $\ddagger$ The comparisons among groups, One-Way ANOVA, a: Group 1 vs Group 2 ( $p=0.006$ ), b: Group 1 vs Group 3 ( $p<0.001$ ), c: Group 2 vs Group 3 $(p<0.001)$.

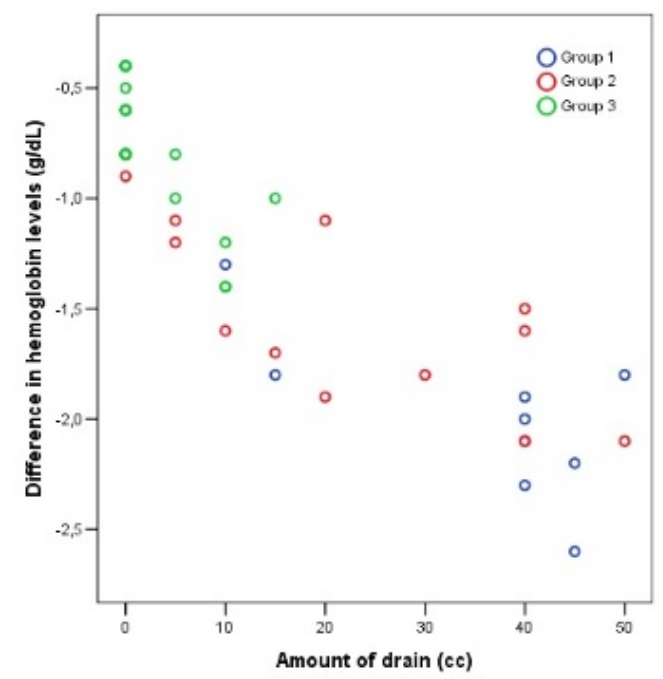

Figure 2. Difference of hb level in groups
Group 1 ( $\mathrm{p}=0.006$ and $\mathrm{p}<0.001$, respectively), and the mean amount of bleeding in Group 3 was found to be statistically significantly lower compared to Group $2(\mathrm{p}<0.001)$ (Figure 1,2) (Table 2).

Upon comparing the patients in the groups in terms of the length of hospitalization, it was observed that patients in Group 3 were discharged statistically significantly earlier than patients in Groups 1 and 2 and patients in Group 2 were discharged statistically significantly earlier than patients in Group $1(\mathrm{p}<0.001)$.

A minor complication (opening at the surgical incision site) developed only in 2 (4.4\%) patients in Group 2. Upon comparing the groups in terms of the rate of incidence of complications, no statistically significant difference was detected between the groups $(\mathrm{p}=0.074)$ (Table 1$)$.

The total fluid amount collected in the drains in the postoperative 24 th hour was measured as $40(10-50) \mathrm{ml}$ on 
Table 3. The coefficients of correlation and significance levels between decrease in hemoglobin levels and amount of drain

\begin{tabular}{lccc}
\hline & $\mathbf{n}$ & Coefficient of correlation & $\mathbf{p}$-value $\boldsymbol{+}$ \\
\hline Group 1 & 9 & -0.533 & 0.139 \\
\hline Group 2 & 13 & -0.767 & $\mathbf{0 . 0 0 2}$ \\
\hline Group 3 & 23 & -0.740 & $<\mathbf{0 . 0 0 1}$ \\
\hline Overall & 45 & -0.922 & $<\mathbf{0 . 0 0 1}$ \\
\hline
\end{tabular}

+ Spearman's rank correlation test.

average in Group 1, 20(0-50) $\mathrm{ml}$ on average in Group 2, and $0(0-50) \mathrm{ml}$ on average in Group 3, and it was determined that this difference between the groups was statistically significant $(\mathrm{p}<0.001)$ (Table 1$)$.

Upon examining the relationship between the amount of fluid comming from the drain in the postoperative 24th hour and the amount of a decrease in the hb value (bleeding) in the postoperative period, no statistically significant correlation was found between the amount of liquid collected in the drain and the amount of change in the hemoglobin level in Group 1 patients $(r=-0.533$ and $\mathrm{p}=0.139$ ); however, the hemoglobin level decreased statistically significantly more as the amount of fluid collected in the drain increased in Group 2 and Group 3 ( $r=-$ $0.767, \mathrm{p}=0.002$ and $\mathrm{r}=-0.740, \mathrm{p}<0.001$ ). Upon comparing all the cases without dividing them into groups, it was determined that the hemoglobin level decreased statistically significant more and more bleeding occurred as the amount of fluid in the drain increased $(r=-0.922$ and $\mathrm{p}<0.001)$ (Table 3).

\section{DISCUSSION}

The aim of the surgical treatment of gynecomastia is to ensure the formation of normal chest contours without contour irregularity and excessive scar tissue $[15,16]$. In the treatment of Simon 1 and 2 cases, liposuction, open resection or the combination of these two are used as the surgical treatment method. In Simon 3 cases, open reduction with skin resection is required $[1,5,6]$.

In the surgical treatment of gynecomastia, particularly, in pseudogynecomastia, in which adipose tissue is predominant, liposuction is the most effective method $[8,15]$. However, in patients with hard or fibrous breast tissue, these tissues cannot be aspirated by a liposuction cannula, and thus, open surgery is required [6]. Traditionally, a periareolar or inframmarial incision is sufficient for subcutaneous mastectomy performed with open surgery [5].

The early complications of surgical treatment are bleeding and hematoma, infection, seroma development, and failure of healing at surgical incision sites [16]. Similarly to the procedures in which bleeding problem is experienced, such as reduction mammoplasty, rhinoplasty, facelift, and liposuction in aesthetic surgery, the infiltration of adrenaline-containing solutions is performed to reduce the amount of bleeding in gynecomastia treatment $[7,8]$.

The increased amount of adrenaline in the tissues leads to a more vasoconstriction effect on blood vessels and less bleeding. Therefore, as the amount of adrenaline increases in infiltration solution increases, the amount of bleeding in the tissue is expected to decrease. The amount of adrenaline collected in the tissue by infiltration is directly proportional to the adrenaline concentration in the infiltration fluid and the amount of fluid used in infiltration. Thus, an increase in the amount of adrenaline can be achieved either by increasing the adrenaline concentration in the solution or by increasing the amount of fluid used in infiltration. It has been shown in many studies that the increase in concentration increases the incidence of side effects $[9,10]$. Thus the only way to increase the amount of adrenaline in the medium without increasing the adrenaline concentration is to increase the volume of solution used in infiltration $[8,12]$.

The first study conducted on the theory that the level of adrenaline in the medium can be increased by increasing the volume of infiltrated fluid and thus, there will be less bleeding was conducted by Beveridge and Bell on patients who underwent reduction mammoplasty, and it was found out that the amount of bleeding decreases as the amount of infiltration solution increases [17]. In the retrospective study conducted on patients who underwent reduction mammoplasty in our clinic, it was revealed that infiltration of above $500 \mathrm{cc}$ significantly reduced the amount of bleeding without increasing the rate of complications, lowered the blood transfusion requirement to a negligible level, and enabled patients to be discharged earlier [8].

In this study, it was revealed that the infiltration of highvolume adrenaline solution reduced the amount of bleeding in patients who received gynecomastia treatment by open surgery. No difference was observed between the groups in terms of the incidence of complications. High volume fluid infiltration and increased amount of adrenaline in the medium reduced bleeding by increasing the vasoconstrictor effect on blood vessels. Furthermore, there are publications reporting that the high volume of fluid applies pressure on the local blood vessels and reduces the amount of bleeding [18]. The amount of fluid collected in the drains of patients in the group in which high volume fluid infiltration was performed was found to be less compared to other groups. Both the reduced amount bleeding and the reduced amount of fluid collected in the drain indicate that the infiltration of high volume adrenaline fluid does not cause rebound bleeding. The less amount of bleeding and fluid collected in the drain ensure that patients are discharged earlier, thus ensuring both lower patient morbidity and lower treatment costs.

In conclusion, it was revealed with the present study that the infiltration of the high volume of adrenaline solution in patients receiving gynecomastia treatment by open surgery 
reduced the amount of bleeding and the amount of fluid collected from the drain without increasing the number of complications and enabled the earlier discharge of patients. Therefore, we recommend high volume fluid infiltration in the surgical treatment of gynecomastia patients.

Declaration of interest: The authors report no conflicts of interest. Financial Disclosure: No financial support was received.

\section{REFERENCES}

1. Erol S, Orhan E, Sevin A, Erdoğan B. Trauma: a new pseudogynecomastia cause. Aesthetic Plast Surg. 2010;34:404-5.

2. Schröder L, Rudlowski C, Walgenbach-Brünagel G, et al. Surgical Strategies in the Treatment of Gynecomastia Grade I-II: The Combination of Liposuction and Subcutaneous Mastectomy Provides Excellent Patient Outcome and Satisfaction. Breast Care (Basel). 2015;10:184-8.

3. Fagerlund A, Lewin R, Rufolo G, et al. Gynecomastia: A systematic review. J Plast Surg Hand Surg. 2015;49:3118.

4. Al-Allak A, Govindarajulu S, Shere $\mathrm{M}$, et al. Gynaecomastia: a decade of experience. Surgeon. 2011;9:255-8.

5. Song YN, Wang YB, Huang R, et al. Surgical treatment of gynecomastia: mastectomy compared to liposuction technique. Ann Plast Surg. 2014;73:275-8.

6. Arvind A, Khan MA, Srinivasan K, Roberts J. Gynaecomastia correction: A review of our experience. Indian J Plast Surg. 2014;47:56-60.

7. Wojnikow S, Malm J, Brorson H. Use of a tourniquet with and without adrenaline reduces blood loss during liposuction for lymphoedema of the arm. Scand J Plast Reconstr Surg Hand Surg. 2007;41:243-9.
8. Orhan E. The efect of high volume adrenalin solution in infiltration on blood loss in reduction mammaplasty. J Clin Anal Med. 2018;9:213-7.

9. Kaplan JL, Rotemberg S, Yetman R, et al. Breast reduction: does the tumescent technique affect reimbursement? Plast Reconstr Surg. 2008; 122: 693700.

10. Hardwicke JT, Jordan RW, Skillman JM. Infiltration of epinephrine in reduction mammaplasty: a systematic review of the literature. Plast Reconstr Surg. 2012; 130: 773-8.

11. Wong KY, Malata CM. Conventional versus ultrasoundassisted liposuction in gynaecomastia surgery: a 13-year review. J Plast Reconstr Aesthet Surg. 2014;67:921-6.

12. Jarrar G, Peel A, Fahmy R, Deol H, Salih V, Mostafa A. Single incision endoscopic surgery for gynaecomastia. J Plast Reconstr Aesthet Surg. 2011;64:e231-6.

13. Sevin A, Senen D, Sevin K, Erdogan B, Orhan E. Antibiotic use in abdominoplasty: prospective analysis of 207 cases. J Plast Reconstr Aesthet Surg. 2007;60:37982.

14. McKee DE, Lalonde DH, Thoma A, Glennie DL, Hayward JE. Optimal time delay between epinephrine injection and incision to minimize bleeding. Plast Reconstr Surg. 2013;131:811-4.

15. Qutob O, Elahi B, Garimella V, Ihsan N, Drew PJ. Minimally invasive excision of gynaecomastia--a novel and effective surgical technique. Ann R Coll Surg Engl. 2010;92(3):198-200.

16. Keskin M, Sutcu M, Cigsar B, Karacaoglan N. Necessity of suction drains in gynecomastia surgery. Aesthet Surg J. 2014;34:538-44.

17. Beveridge $M$, Bell $M$. The tumescent technique for bloodless breast reduction. Can J Plast Surg. 1994; 2: 121-4

18. Conroy PH, O’Rourke J. Tumescent anaesthesia. Surgeon. 2013; 11: 210-21. 